University of Nebraska - Lincoln

DigitalCommons@University of Nebraska - Lincoln

Anthony Zera Publications

Papers in the Biological Sciences

February 2007

\title{
The cost of reproduction: the devil in the details
}

Lawrence G. Harshman

University of Nebraska - Lincoln, Iharshman1@unl.edu

Anthony J. Zera

University of Nebraska - Lincoln, azera1@unl.edu

Follow this and additional works at: https://digitalcommons.unl.edu/bioscizera

Part of the Microbiology Commons

Harshman, Lawrence G. and Zera, Anthony J., "The cost of reproduction: the devil in the details" (2007). Anthony Zera Publications. 6.

https://digitalcommons.unl.edu/bioscizera/6

This Article is brought to you for free and open access by the Papers in the Biological Sciences at DigitalCommons@University of Nebraska - Lincoln. It has been accepted for inclusion in Anthony Zera Publications by an authorized administrator of DigitalCommons@University of Nebraska - Lincoln. 
Published in Trends in Ecology \& Evolution 22:2 (February 2007), pp. 80-86. doi:10.1016/j.tree.2006.10.008 Copyright (c) 2006 Elsevier Ltd. Used by permission. Published online October 23, 2006.

\title{
Review
}

\section{The cost of reproduction: the devil in the details}

\author{
Lawrence G. Harshman* and Anthony J. Zera \\ School of Biological Sciences, University of Nebraska, Lincoln, NE 68588, USA \\ *Corresponding author. Email: Iharsh@unlserve.unl.edu
}

\begin{abstract}
The cost of reproduction is of fundamental importance in life-history evolution. However, our understanding of its mechanistic basis has been limited by a lack of detailed functional information at all biological levels. Here, we identify, evaluate and integrate recent studies in five areas examining the proximate mechanisms underlying the cost of reproduction. Rather than being alternate explanations, hormonal regulation and intermediary metabolism act in concert and have an overarching influence in shaping the cost of reproduction. Immune function is compromised by reproduction, as is resistance to environmental stress. These studies not only provide new information about mechanisms that comprise "the cost," but also hint at an underlying evolutionarily conserved causal mechanism.
\end{abstract}

\section{Introduction}

The demographic cost of reproduction, (i.e. decreased survival and future reproduction as a function of present reproduction), is a pivotal tradeoff around which life histories are thought to evolve $\left[\begin{array}{ll}1 & \&\end{array} 2\right]$ and, owing to its fundamental importance, has been of interest to researchers from a variety of biological fields $[2,3,4,5,6, \& 7]$. The functional causes of the cost have been a long-standing issue in evolutionary biology $[4,5,8, \& 9]$, and the traditional view is that internal energy reserves are limited, thus giving rise to tradeoffs; in particular, current reproduction reduces future reproduction and survival. More recently, an alternate view has emerged, in which "pleiotropic" effects of regulators cause the cost [7]. These opposing views highlight a major problem in the field, namely the lack of detailed information about the proximate mechanisms necessary to evaluate competing functional explanations of the demographic cost of reproduction.

Recently, there has been an increase in the number of studies investigating the proximate mechanisms underlying the cost of reproduction. Here, we review what we consider to be five areas in which significant information has been obtained regarding these mechanisms: (i) hormonal regulation; (ii) intermediary metabolism and allocation; (iii) immune function; (iv) reproductive proteins; and (v) defenses against stress and toxicity. Regulation and metabolism are viewed as overarching factors that shape the cost of reproduction, whereas compromised immune function and decreased protection against stress and toxicity are viewed as proximate effectors of the cost. Much of this new information comes from studies on invertebrates, which are our primary focus here.

\section{Five components of the cost of reproduction}

\section{Hormonal regulation}

Because of their many "pleiotropic" (i.e. multiple) effects, hormones have long been thought to be key mediators of the cost of reproduction and life-history tradeoffs $[4,5,9, \& 10]$. Until recently, most endocrine work on life histories was vertebrate-based and focused on phenotypic associations between reproductive hormone levels and various costs of reproduction, most notably reduced adult survivorship $[4,5,9,11$, \& 12]. Recent investigations using Drosophila mutations and artificial selection in wing-polymorphic crickets, such as Gryllus firmus, are now providing the first detailed information about the evolutionary genetics of life-history tradeoffs. Three hormone systems are the foci of these studies: (i) insulin signaling (Box 1 and Box 2); (ii) juvenile hormone (JH); and (iii) ecdysteroid signaling, the latter two of which regulate many aspects of reproduction.

Mutant Drosophila melanogaster (see Box 3 for a discussion of mutation analysis of the cost of reproduction) that have reduced insulin signaling can be long-lived as well as dwarf (about half their normal size) and female sterile [13 \& 14]. In female flies, mutations in the gene encoding the Drosophila insulin receptor $(D I n R)$ result in a relatively low rate of ecdysteroid release from the ovaries in vitro [15] and reduced JH biosynthesis in vitro compared with wild-type females [13]. Tatar et al. [13] concluded that defective insulin signaling resulted in decreased $\mathrm{JH}$ biosynthesis and systemic JH titer, which, in turn, suppressed egg production and increased longevity. Low levels of JH biosynthesis in vitro were also observed in individuals with a mutation in chico, a gene- 


\section{Box 1. An introduction to insulin/insulin-like growth factor signaling}

Insulin/insulin-like growth factor signaling regulates many aspects of intermediary metabolism in addition to processes ranging from growth to aging. Insulin is a peptide hormone produced in the mammalian pancreas and insulin-like peptides are found in invertebrates. Insulin is secreted into the circulatory system and interacts with a receptor that is found on the surface of most cells. The insulin receptor mediates signals in response to external effectors and intracellular states by interaction with an insulin substrate protein (IRS-1 in humans). The substrate protein initiates phosphorylation that activates a kinase cascade (enzymes that add a phosphate group). An important downstream target of the signaling cascade is a transcription factor that regulates the expression of many genes. From Caenorhabditis to Homo, this transcription factor is part of the forkhead transcription factor family (FOXO).

The essential components of insulin signaling in $C$. elegans are insulin-like ligands, the insulin receptor DAF-2, (encoded by daf$2)$, the kinase cascade (PI3K, akt/PKB), and the FOXO transcription factor DAF-16 (encoded by daf-16). Insulin and insulin-like peptides constitute systemic messengers, which activate DAF-2. This sets off a signaling cascade of sequential phosphorylation of regulatory proteins (e.g. PI3K and akt/PKB). When a downstream transcription factor, DAF-16, is phosphorylated, it does not translocate to the nucleus, remaining instead in the cytoplasm [66]; this influences the expression of many genes that, in aggregate, negatively affect life span [66]. At low levels, or in the absence of insulin signaling, DAF-16 remains unphosophorylated and translocates to the nucleus, binding to many sites across the genome, and an ultimate effect of altered gene expression is increased life span [67 \& 68].

The range of insulin signaling effects on physiology stems partly from interactions with other pathways, such as the intracellular TOR (target of rapamycin) pathway that regulates protein biosynthesis. Insulin and TOR signaling are known to interact to regulate growth, metabolism and life span [69 \& 70].

encoding an insulin substrate protein [16]. However, in a different study [17], flies with the same mutation exhibited neither reduced JH biosynthesis nor lowered levels of ecdysteroids [17]. The differences between these studies (Refs 13 and 16 versus Ref. 17) could be due either to the specific gene that was mutated in the insulin signaling pathway ( $D I n R$ versus chico) or to genetic background. However, the only functional test in this series of studies (reciprocal ovarian transplants between wild-type and chico $^{l}$ females) showed that wild-type ovaries produced mature eggs in chico $^{l}$ females, whereas chi$c o^{l}$ ovaries did not mature in wild-type individuals [17]. Thus, the systemic factors necessary for egg production are present in homozygous chico $^{l}$ individuals. This also showed that the effect of the chico $^{l}$ mutation on egg maturation was confined to the ovary (i.e. this is not a systemic endocrine effect; also see Ref. [18]). In general, genetic evidence indicates that, in Drosophila, at least one hormonal system (insulin signaling) has an important role in the demographic cost of reproduction, whereas the role of $\mathrm{JH}$ is an open question.

Naturally occurring genetic covariation among hormone levels and components of the tradeoff between reproduction and flight capability has been reported for artificially selected lines of wing-polymorphic crickets [19]. In G. firmus, earlyage fecundity, the hemolymph ecdysteroid titer and the hemolymph JH titer were all elevated in adults of SW (short-wing) compared with LW (long-wing) selected lines [19 \& 20]. These results indicate that hormones mediate this cost of reproduction by linking positive effects of enhanced reproduction with negative effects on somatic function (i.e. ability to disperse). Even more dramatic was the morph-specific circadian cycle in the $\mathrm{JH}$
Box 2. Reproductive system signals that affect the life span of Caenorhabditis elegans: rethinking the rethinking

Recent studies of lifespan in C. elegans have given rise to the hypothesis that signals from the reproductive system influence longevity without significantly altering internal resource allocation [7]. For example, RNAi suppression of insulin receptor gene (daf-2) function (Box 1) from shortly after adult emergence through to the end of adult life extended longevity, but did not alter fecundity; neither did ablation of the entire gonad (germ line and somatic gonad) affect life span [61]. These results are surprising given that removal of a reproduction sink (i.e. egg production) for resources does not necessarily free those resources for somatic function that extends longevity, as would be expected from the traditional view of the $Y$ model of resource allocation. Even more surprising, specific ablation of only the germ line extends life span, whereas specific ablation of the somatic gonad reduces it [61]. This work has collectively given rise to what has been described as a new hypothesis regarding the cost of reproduction: signals emanating from the reproductive system have a direct negative effect on survival, rather than the classic view that reproduction diverts energy from somatic functions [7]. However novel, this hypothesis [7] is consistent with earlier endocrine studies in vertebrates (i.e. testosterone as an immunosupressant) $[4,5$ \& 11$]$.

There is an alternate interpretation of the cause of the negative effect of the germ line signal on survival. This signal depends on the presence of a functioning insulin receptor (encoded by a wildtype allele of daf-2), an insulin signaling nuclear hormone receptor (daf-12) and a functional daf-16, which is the downstream transcription factor controlled by the insulin signaling cascade [71] (Box 1). In other words, the negative effect of signals from the reproductive system depends on a functional insulin signaling pathway, which is known to regulate many aspects of metabolism. This observation raises the question as to whether the mode of action of the life spandecreasing signal from the germ line is due to altered somatic anabolism. If this is correct, then there is no clear dichotomy between signaling as opposed to altered metabolism as a cost of reproduction. The issue is complex and can only be resolved by functional studies at multiple levels of biological organization in the context of life-history trait variation.

titer [19 \& 21]: on a daily basis during adulthood, the JH titer in the LW morph rises above and then drops below the temporally invariant $\mathrm{JH}$ titer in the SW morph. This pattern suggests that the hormonal control of a cost of reproduction is regulated not only by the level of $\mathrm{JH}$, but also by the duration of time that the $\mathrm{JH}$ titer is elevated above some threshold.

These studies underscore the importance of extensive in vivo characterizations of hormone titers in studies of the cost of reproduction. Hormonal regulation is also an important factor in each of the four aspects of the cost of reproduction discussed below, as well a global organizer of the cost itself.

\section{Intermediary metabolism and allocation tradeoffs}

A core idea in life-history physiology is that the differential allocation of limited internal resources (the traditional " $Y$ " model of resource allocation, Figure 1a) has a central role in the cost of reproduction and other life-history tradeoffs $[3,4,5 \&$ 10]. However, until recently, little was known about the causal mechanisms involved, such as changes in the flow of metabolites through pathways of intermediary metabolism. Recent biochemical and metabolic studies on allocation tradeoffs in wing-polymorphic crickets have provided detailed information about the functional causes of a resource-based allocation tradeoff that underlies a key life-history tradeoff [4 \& 22].

In lines of G. firmus artificially selected for the flightless (SW) versus flight-capable (LW) morph, early-age fecundity trades off with dispersal capability: SW females exhibit a 
Box 3. Mutation analysis of the cost of reproduction

One of the most important techniques recently used to investigate the cost of reproduction is mutation analysis, a widespread approach in contemporary genetics. A good example of the power and limitations of mutational analysis in the context of the cost of reproduction is analysis of the INDY mutation in D. melanogaster.

An induced mutation (allele) at the INDY gene causes a surprising relationship among traits: homozygous mutant females exhibit increased longevity without lowered fecundity [72]. The mutation circumvents the negative relationship between life span and reproduction. INDY encodes a tranporter that moves metabolites into the mitochondrion and thus points to mitochondrial metabolism as a contributor to the cost of reproduction in wild-type individuals. An argument could be made from mutational analysis of lifespan that there is no physiological, developmental, or other endogenous constraint that prevents the decoupling of reproduction and survival. For example, there appears to be little, if any, survival cost of egg production in C. elegans [7], which implies that egg production does not constrain survival. However, such arguments are problematic. Mutations of large effect such as INDY (and mutations in the insulin signaling pathway), might disrupt the normal physiology of the organism so much that they result in an aberrant condition in which constraints do not operate, or exert their effects, in a normal manner. In the case of INDY and especially the daf mutations (Box 1), metabolism is profoundly altered and the normal consequences of anabolism and catabolism are at least partly removed. The method of mutational analysis used to study the phenomena of interest (costs of reproduction) might impact the organism to such a degree that the pleiotropic effects remove normal trait relationships.

$100-400 \%$ enhancement of ovarian growth and a $30-40 \%$ reduction in somatic triglyceride (flight-fuel) reserves compared with LW females. Because both morphs consume and assimilate an equivalent amount of resources, morph differences in somatic and reproductive reserves are not due to variation in nutrient input, but to variation in internal allocation [4].

In vivo radiotracer studies in G. firmus demonstrate that the LW morph diverts a proportionately greater amount of fatty acids and amino acids to the biosynthesis of triglyceride flight fuel and a proportionately lower amount to the biosynthesis of ovarian protein compared with the high-fecundity SW morph [22, $23 \& 24]$. These studies show that nutrients flow differentially through bifurcating pathways of metabolism in phenotypes that differ in life histories, an important aspect of the "Y" model of internal resource allocation. Morph-specific changes in pathway flux result from large-scale changes in the activities of multiple enzymes of metabolism [22 \& 25]. This is consistent with theoretical studies of metabolism [26] and with results of laboratory selection for metabolic efficiency in yeast [27], which indicate that the activities of many enzymes must be altered to change the flow of nutrients through a metabolic pathway.

Limited internal resources have typically been viewed as the constraint that causes allocation tradeoffs [4]. However, endocrine studies in G. firmus suggest that hormonal regulation also has a crucial role. A JH analog applied to LW females caused the expression of biochemical (e.g. reduced triglyceride biosynthesis) and reproductive traits (increased egg production) typically seen in SW morphs [22 \& 23]. Hormonal control of metabolic tradeoffs is not surprising, given the tight hormonal control of flux through pathways of intermediary metabolism (see citations in Ref. [25]). However, negative pleiotropic effects of hormones vs. limited internal resources have often been proposed as alternate explanations for life-history tradeoffs [7]. By contrast, stud-

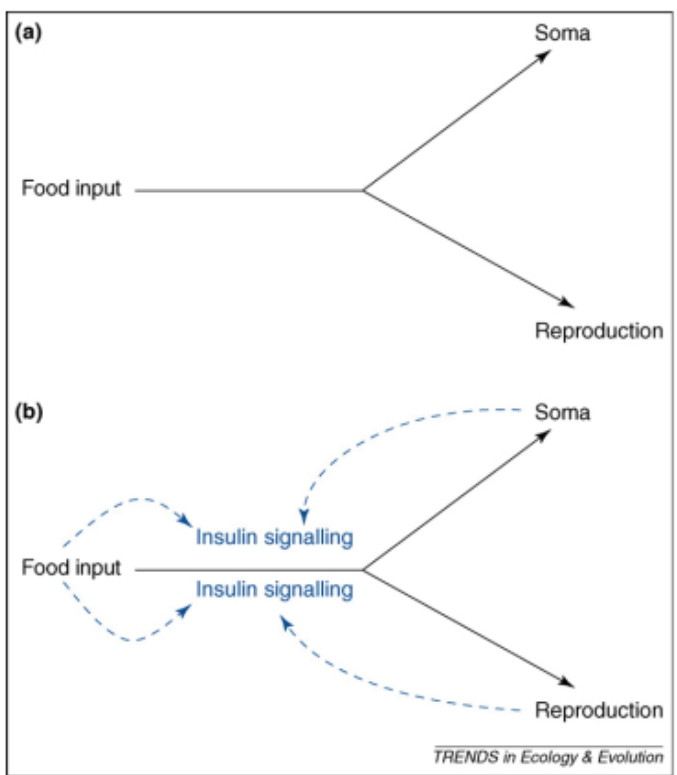

Figure 1. $\mathbf{Y}$ models of the cost of reproduction. The traditional " $Y$ " model of resource allocation (a) is the framework of various lifehistory trait tradeoffs. Many models and empirical studies have been based on this framework. Food input is shown at the base of the "Y" and energy resources are allocated to reproduction versus the rest of the body (soma). The apportionment to reproduction, at the expense of limited resources available for the soma, forms the basis of the cost of reproduction. In (b), a hypothetical role is shown for insulin signaling as an integrator of signals from the endpoints of the $Y$ model. It provides the information needed to adjust or maintain differential resource allocation to either reproduction or the soma. Signals about food availability and input, as well as feedback from the reproductive system and the rest of the body (i.e. soma) are represented by blue dotted lines. Although the key role of insulin signaling in mediating the relationship between survival and reproduction has been recognized (e.g. Refs $13 \& 54$ ), this model emphasizes a different aspect of insulin signaling, which is to integrate and allocate resources. The cost of reproduction is caused by insufficient energy being allocated to the soma to protect it from damaging agents (e.g. pathogens, environmental stress, toxic metabolites, etc.).

ies in G. firmus suggest that variation in endocrine control interacts with metabolism to mediate differential allocation.

Analogous in vivo radiotracer studies in lepidopterans are beginning to identify the detailed proximate mechanisms of aminoacid allocation to egg protein biosynthesis [28, $29 \& 30]$, resulting in new insights into the metabolic constraints on life-history evolution. For example, egg production in species whose adults primarily feed on nectar (i.e. primarily sugar) might be more constrained by their inability to synthesize the complex carbon skeletons that make up essential amino acids than by the availability of nitrogen per se, the factor most commonly thought to limit egg production in plant-feeding insects [28, $29 \& 30]$.

\section{Immune function}

The tradeoff between reproduction and immune function is one of the most intensively investigated aspects of the cost of reproduction, mainly in male birds. Several studies have documented that increased reproductive effort is associated with either increased susceptibility to parasitism and disease [31] or decreased immune system function [4, $5 \& 32]$. Competition between reproductive and immune functions for limited inter- 
nal energy reserves, or negative "pleiotropic" effects of reproductive hormones (e.g. testosterone) on immune function, have been proposed as proximate causes of the cost of reproduction. However, the mechanisms involved are poorly understood even in the best-studied cases. Contradictory results are common [18 \& 33], which might be due to the diversity of measures of immune function that have been used, variation in experimental conditions, or other factors, such as the physiological state of the subject. Another concern is defining the "optimal" immune response [34], which, for example, makes it difficult to determine whether an elevated immune response is adaptive or maladaptive. Viney et al. [34] advocate defining an appropriate immune response in terms of how the response affects fitnessrelated (e.g. life-history) traits, which is not usually done.

With the above problems in mind, there is evidence from vertebrate and invertebrate studies that elevated immune function extracts a fitness cost [34 \& 35]. Some evidence indicates an energetic cost, whereas direct somatic damage by the immune response could also be responsible for the impact on life-history traits such as survival.

Invertebrate model systems are ideal for investigating the tradeoff between reproduction and immune function, including measurement of relevant genetic correlations [36]. For example, a study of the ground cricket Allonemobius socius found that an increased number of matings was associated with suppressed immune function and decreased longevity [37]. Manipulation of mating status and diet revealed that female $D$. melanogaster lose immune function under conditions of food limitation, whereas males exhibit reduced immune responses as a function of sexual activity [38]. Recent evidence suggests that pleiotropic effects of JH link immune function and reproduction. In the flour beetle Tenebrio molitor, mating was reported to cause immunosuppression (decreased phenoloxidase activity) by increasing the level of $\mathrm{JH}$ [39]. These results are consistent with the hypothesis that $\mathrm{JH}$ reduces immune function. However, a limitation of this study is that the JH titer was not measured directly. Recently, behavioral manipulation of honey-bee Apis mellifera workers increased the $\mathrm{JH}$ titer (measured directly by radioimmunoassay) and increased longevity, but decreased immune function [40]. Interestingly, in this [40] and other studies [41 \& 42], a higher level of immune function was negatively associated with survival. Thus, the effect of immune function on survival is likely to be double-edged and contingent on both intrinsic and environmental conditions.

\section{Drosophila male accessory gland proteins}

In various insect taxa, male proteins transferred to females during mating negatively affect female fitness, thus constituting a cost of reproduction [43]. The proximate mechanisms underlying this cost of mating are best understood in D. melanogaster. The main players are male accessory gland proteins (Acps). Approximately $80 \mathrm{Acps}$ are transferred to $D$. melanogaster females during mating, where they exert a range of physiological effects [44 \& 45]. Experiments using males from a transgenic line in which the main cells of the male accessory gland (the source of Acps) had been ablated by toxin expression, showed that Acps are largely responsible for the cost of mating [46].
The effect of each of eight Acps on female survival was tested by expressing the corresponding genes in transgenic females [47] and one of these, Acp62F, was shown to reduce female survival. Acp62F encodes a protease inhibitor $90 \%$ of which remains in the female reproductive tract after transfer from males, but $10 \%$ of which enters the circulatory system, where it could interfere with the normal function of non-target proteases. Thus, a specific protein might be responsible for increased mortality as an inadvertent byproduct of escape from the female reproductive tract. This could contribute to the cost of reproduction by a mechanism that is independent of metabolism or regulation, the two mechanisms that are generally thought to underlie the cost of reproduction.

Acp70 (sex peptide) is an important contributor to the cost of mating [48]. The sex peptide, covalently bound to sperm, is transferred to females at the time of mating. It is cleaved from sperm [49], enters the female circulatory system and strongly stimulates egg production in addition to suppressing female remating. It also stimulates the biosynthesis of JH [50] which is thought to be the basis for increased vitellogenesis (yolk deposition) and elevated egg production after mating. The effect of the sex peptide on females is complex partially because it appears to involve modulation of a global endocrine regulator $(\mathrm{JH})$ that is associated with a range of physiological effects, including altered immune function and metabolism. The interaction between Acps and endocrine regulation highlights the interactions among the various components of the cost of reproduction.

In addition to being a model to investigate the cost of reproduction, Acps also show promise for investigating sexual conflict, which has been defined as antagonism between the evolutionary interests of the two sexes [51]. Sexual conflict is an important new topic in evolutionary biology [52] with profound implications for life-history evolution [53]. The relevance of Acps stems from the paucity of information about proximate mechanisms underlying the evolutionary consequences of sexual conflict, the rapid increase in detailed insight into the mode-of-action of Acps and the possibility that sexually antagonistic effects of Acps have evolved in the context of sexual conflict. Studies of Acps could thus link research on sexual conflict with that on the cost of reproduction.

\section{Defense against stress and toxicity}

There are two scenarios under which reproduction could result in decreased survival after exposure to a variety of stressful, toxic, or otherwise damaging conditions. The first scenario is that reproduction results in direct somatic damage, causing increased vulnerability to environmental stress, toxins and other damaging factors. The second is that reproduction drains somatic energy reserves (e.g. carbohydrate and lipid), thus limiting the energy available for biochemical systems that protect the body from damage. Some detoxification systems are energetically expensive [54] and tradeoffs might be mediated by "inadequate resource investment in somatic maintenance" [54] given the costs involved in detoxification and resistance to stress. Relevant stress and/or damaging conditions could include heat, cold, oxidation, starvation, extrinsic toxins, intrin- 
sic metabolites that have toxic (damaging) effects, endoplasmic reticulum stress, osmotic stress, and so on. However, only a few studies, using D. melanogaster and zebra finches Taeniopygia guttata, have addressed directly the issue of whether susceptibility to stress or toxins is a cost of reproduction.

Phenotypic manipulations in D. melanogaster have been used to elevate egg production followed by monitoring adult survival under stressful conditions [55, $56 \& 57]$. Two studies investigated the effects of treatments known to stimulate egg production (i.e. a JH analog, mating and live yeast) on subsequent survival under oxidative stress and starvation conditions using fertile wildtype strains and a sterile female mutation strain [56 \& 57]. The treatments decreased survival under oxidative stress and sometimes under starvation conditions in wild-type strains relative to untreated fertile females. In a similar experiment, mating, or adding yeast, decreased the survival of fertile females relative to untreated fertile females when both were raised in a chamber with high oxygen content; by contrast, sterile mutant females were not differentially affected by the mating and added yeast treatments (Y. Wang, PhD Thesis, University of Nebraska 2005). There was also evidence for oxidative damage to proteins as a cost of mating (Y. Wang, PhD Thesis, University of Nebraska 2005). Oxidative stress damage to macromolecules is particularly compelling as a candidate for a cost of reproduction partly because it is related to the free radical theory of aging, which posits that oxidative damage to macromolecules reduces survival [58].

Manipulations of zebra finch brood size have been followed by assays to monitor the effects on adults in terms of oxidative stress susceptibility or antioxidant defense [59 \& 60]. In one study [59], brood size was manipulated by varying the number of nestlings. Whole blood was collected from the adults and incubated with an oxidant that causes lysis of the red blood cells. Increased lysis was reported as a function of reproductive effort of the adults, suggesting that the antioxidant system of the adult bird was compromised. In another study based on experimental enlargement of brood size [60], the decreased activity of glutathione peroxidase and superoxide dismutase (both antioxidant enzymes) was reported in adult females and males when enzyme activity was scaled to daily energy expenditure.

\section{Conclusion}

Here, we have identified recent advances in our understanding of the mode-of-action of five key components of the cost of reproduction (regulatory signals; intermediary metabolism; immune function; accessory gland proteins as examples of reproductive proteins that potentially exert sexually antagonistic effects; stress and toxicity). These categories comprise upstream global mediators (regulation and metabolism, Box 4) and downstream effectors of the cost. An emerging unifying theme is the overriding importance of metabolism (anabolism and catabolism) and hormonal regulation as key players in the cost of reproduction (Box 4).

\section{Proximate effectors of the cost of reproduction}

The proximate effectors of the cost of reproduction include compromised immune function as well as decreased defense
Box 4. The " $Y$ " network model of the cost of reproduction

We consider metabolism and regulatory signals to be key upstream global mediators of the cost of reproduction. Systemic regulators of metabolism, such as insulin signaling, could control energy allocation to somatic and reproductive functions. In other words, nutrient sensing, nutrient input and feedback form the endpoints of resource allocation (i.e. the soma and reproduction), are likely to adjust insulin signaling, which, in turn, modifies pathway input and output (allocation; Figure 1b, main text).

In addition, insulin signaling could interact with other pathways of metabolism to mediate allocation. For example, TOR signaling responds to amino acid concentration and is part of the nutrient sensing system. It regulates protein biosynthesis and is a prime candidate to regulate allocation in conjunction with insulin signaling. Importantly, insulin signaling is systemic and, thus, is most suited to the role of the key integrator of signals regarding food, reproduction and somatic state, and also to have the lead role in mediating the cost of reproduction by controlling differential allocation.

In terms of the " $Y$ " network model of the cost of reproduction, there are signaling points at the input (nutrient sensing and food uptake) and at the outputs (somatic state and function, level of reproduction). Our hypothesis is that the cost of reproduction is mediated by differential energy allocation to reproduction or somatic function under control of insulin signaling, which responds to signals of the three points of the network described above. This viewpoint differs from the hypothesis (Box 2) that signaling from the reproductive system reduces survival independent of metabolism. Our interpretation is that the signals from the reproductive system (Box 2) are a means of communicating the appropriate level of energy for reproduction and that the metabolic impact on the rest of the body affects survival. The cost of reproduction is a function of metabolism and regulation, or rather, the interplay between the two.

against stress. Reproduction might drain energy from somatic stores, resulting in a decreased capacity to mount an effective immune response or to produce enough protein, or other molecules, to protect against damage from stress and toxicity. Alternatively, reproduction acting through metabolism might be damaging (e.g. producing damaging reactive oxygen species). It is not clear whether altered metabolism causes the cost of reproduction because of a shortage of energy for somatic maintenance and defense, because of damaging byproducts of metabolism, or because of a combination of compromised defense systems exacerbated by generation of damaging agents.

\section{Future studies}

An important recent advance in studies on the cost of reproduction is the identification of specific pathways that potentially underlie such costs. Examples include metabolic branch points in lipid and amino acid metabolism [22, $23 \& 24]$ and the insulin signaling pathway [ $6 \& 61]$. One of the most important goals of future studies should be to identify and investigate additional pathways that are likely to contribute to the cost of reproduction, such as those underlying innate immunity or detoxification of exogenous, or endogenously generated, compounds that can cause biological damage.

Environmental variation and natural or laboratory populations should be included in studies on the cost of reproduction. For example, the INDY mutation in D. melanogaster (Box 3) exhibits a nutrient-dependent cost of reproduction [62]. Food availability and input are likely to be among the most important environmental influences on the cost of reproduction, and input is a crucial feature of the Y model of re- 
source allocation [4 \& 63]. Selection studies using laboratory populations are important for putting mutational analysis of the cost of reproduction in a population-genetic context. For example, selection for extended longevity and late-age reproduction using $D$. melanogaster has resulted in decreased early age reproduction in five out of six studies (summarized in Ref. [64]). These results, produced by independent selection experiments, provide evidence for a general evolutionary outcome whereby reproduction and survival are negatively correlated. This genetic correlation is based on genetic variation that is thought to reflect variation segregating in natural populations. The robust negative genetic correlation contrasts with the observation that major effect mutations can "break" the cost of reproduction (Box 3). In general, the cost might be due to an evolved phenomenon ensuing from cumulative allelic effects under selection rather than to physiological or structural constraints between the traits that can be decoupled by mutation. Unfortunately, the genes that mediate the cost of reproduction in laboratory or natural populations are currently unknown.

An additional challenge is to understand the extent to which the players underlying the cost of reproduction interact to exert their effects. For example, do insulin and TOR signaling (Box 1) interact to mediate differential allocation that controls the cost of reproduction?

Intriguingly, recent studies hint at an evolutionarily conserved mechanism underlying the cost of reproduction. If a general explanation exists, then it might be similar to the hypothesis presented in Box 4 and Figure 1. Only time and future studies on proximate mechanisms of the cost of reproductions will enable rigorous tests of this hypothesis.

An increasing number of evolutionary biologists consider detailed information on proximate mechanisms essential for a better understanding of evolutionary phenomena, including the cost of reproduction [4, $6 \& 65]$. Using West-Eberhard's analogy [65], if we treat a phenomenon such as the cost of reproduction as a black box, then the players and processes that inhabit it will be imaginary constructs that might have little connection to reality. We will never understand the consequences of the cost of reproduction on ecological and evolutionary processes, or how these processes shape "the cost," unless we understand its mechanistic basis, to the point of prediction about how the box operates in different ecological and evolutionary contexts. Indeed, the devil is in the details.

\section{Acknowledgments}

This review was supported by an Army Research Office grant (1R24 GM65513) to L.G.H. and National Science Foundation grants to A.J.Z. (IBN0130665, IBN-0212486 and IOB-0516973). We thank Eileen Hebets for valuable comments; Mariana Wolfner and Tracey Chapman for valuable input on the Drosophila accessory gland protein section; and Christine Pauls for timely assistance and care in preparing the figure. This work is dedicated to George Williams for insight into the central role of the cost of reproduction in evolution and, in general, acute insight into how life histories evolve.

\section{References}

1 G.C. Williams, Natural selection, the costs of reproduction, and a refinement of Lack's principle, Am. Nat. 100 (1966), pp. 687-690.

2 S.C. Stearns, The Evolution of Life Histories, Oxford University Press (1992).
3 C.R. Townsend and P. Calow, Physiological Ecology. An Evolutionary Approach to Resource Use, Blackwell Scientific Publications (1981).

4 A.J. Zera and L.G. Harshman, Physiology of life history trade-offs in animals, Annu. Rev. Ecol. Syst. 32 (2001), pp. 95-126.

5 T. Williams, Mechanisms underlying the costs of egg production, BioScience 55 (2005), pp. 39-48.

6 L. Partridge et al., Sex and death: what is the connection?, Cell 120 (2005), pp. 461-472.

7 A.M. Leroi, Molecular signals versus the Loi de Balancement, Trends Ecol. Evol. 16 (2001), pp. 24-29.

8 M.R. Rose and T.J. Bradley, Evolutionary physiology of the cost of reproduction, Oikos 83 (1998), pp. 443-451.

9 E.D. Ketterson and V. Nolan Jr., Adaptation, exaptation, and constraint: a hormonal perspective, Am. Nat. 154 (1999) (Suppl.), pp. S4-S25.

10 R.E. Ricklefs and M. Wikelski, The physiology/life-history nexus, Trends Ecol. Evol. 17 (2002), pp. 462-468.

11 J.C. Wingfield et al., Avoiding the 'costs' of testosterone: Ecological bases of hormone-behavior interactions, Brain Behav. Evol. 57 (2001), pp. 239-251.

12 W. Reed et al., Physiological effects on demography: a long-term experimental study of testosterone's effects on fitness, Am. Nat. 167 (2006), pp. 667-683.

13 M. Tatar et al., A mutant Drosophila insulin receptor homolog that extends life-span and impairs neuroendocrine function, Science 292 (2001), pp. $107-110$.

14 D. Clancy et al., Extension of lifespan by loss of CHICO, a Drosophila insulin receptor substrate protein, Science 292 (2001), pp. 104-106.

$15 \mathrm{M}$. Tu et al., Impaired ovarian ecdysone synthesis of Drosophila melanogaster insulin receptor mutants, Aging Cell 1 (2002), pp. 158-160.

$16 \mathrm{M}$. Tu et al., Mutations in insulin signaling pathway alter juvenile hormone synthesis in Drosophila melanogaster, Gen. Comp. Endocrinol. 142 (2005), pp. 347-356.

17 D. Richard et al., Insulin signaling is necessary for vitellogenesis in Drosophila melanogaster independent of the roles of juvenile hormone and ecdysteroids: female sterility of the chico ${ }^{l}$ insulin signaling mutation is autonomous to the ovary, J. Insect Physiol. 51 (2005), pp. 455-464.

18 D. Drummond-Barbosa and A.C. Spradling, Stem cells and their progeny response to nutritional changes during Drosophila oogenesis, Dev. Biol. 231 (2001), pp. 265-278.

19 A. Zera, Evolutionary genetics of juvenile hormone and ecdysteroid regulation in Gryllus: A case study in the microevolution of endocrine regulation, Comp. Biochem. Physiol. A 144 (2006), pp. 365-379.

20 A.J. Zera and J. Bottsford, The endocrine-genetic basis of life-history variation: relationship between the ecdysteroid titer and morph-specific reproduction in the wing-polymorphic cricket, Gryllus firmus, Evolution 55 (2001), pp. 538-549.

21 Z. Zhao and A.J. Zera, The hemolymph JH titer exhibits a large-amplitude, morph-dependent, diurnal cycle in the wing-polymorphic cricket, Gryllus firmus, J. Insect Physiol. 50 (2004), pp. 93-102.

22 A.J. Zera, Intermediary metabolism and life history trade-offs: Lipid metabolism in lines of the wing-polymorphic cricket, Gryllus firmus, selected for flight capability vs. early age reproduction, Integr. Comp. Biol. 45 (2005), pp. 511-524.

23 Z. Zhao and A.J. Zera, Differential lipid biosynthesis underlies a tradeoff between reproduction and flight capability in a wing-polymorphic cricket, Proc. Natl. Acad. Sci. U. S. A. 99 (2002), pp. 16829-16834.

24 A.J. Zera and Z. Zhao, Intermediary metabolism and life-history trade-offs: differential metabolism of amino acids underlies the dispersal-reproduction trade-off in a wing-polymorphic cricket, Am. Nat. 167 (2006), pp. 889-900.

25 A. Zera and Z. Zhao, Life-history evolution and the microevolution of intermediary metabolism: activities of lipid-metabolizing enzymes in life-history morphs of a wing-dimorphic cricket, Evolution 57 (2003), pp. 568-596. 
26 D. Fell, Understanding the Control of Metabolism, Portland Press (2003).

27 T. Ferea et al., Systematic changes in gene expression patterns following adaptive evolution in yeast, Proc. Natl. Acad. Sci. U. S. A. 96 (1999), pp. 9721-9726.

28 C.L. Boggs, Dynamics of reproductive allocation from juvenile and adult feeding: radiotracer studies, Ecology 78 (1997), pp. 192-202.

29 D.M. O'Brien et al., Renewable and nonrenewable resources: Amino acid turnover and allocation to reproduction, Proc. Natl. Acad. Sci. U. S. A. 99 (2002), pp. 4413-4418.

30 D.M. O'Brien et al., The amino acids used in reproduction by butterflies: a comparative study of dietary sources using compound-specific stable isotope analysis, Physiol. Biochem. Zool. 78 (2005), pp. 819-827.

31 K. Norris and M.R. Evans, Ecological immunology: life history trade-offs and immune defense in birds, Behav. Ecol. 11 (2000), pp. 19-26.

32 I.P.F. Owens and K. Wilson, Immunocompetence: a neglected life history trait or conspicuous red herring?, Trends Ecol. Evol. 14 (1999), pp. $170-172$.

33 C.G. Greenman et al., Reproductive state, but not testosterone, reduces immune function in male house sparrows (Passer domesticus), Physiol. Biochem. Zool. 78 (2005), pp. 60-68.

34 M. Viney et al., Optimal Immune responses: immunocompetence revisited, Trends Ecol. Evol. 20 (2005), pp. 665-669.

$35 \mathrm{~J}$. Rolff and M. Siva-Jothy, Invertebrate immunity and limits of mechanistic immunology, Nat. Immunol. 6 (2003), pp. 651-654.

36 Lawniczak, M.K.N. et al. (2007) Mating and immunity in invertebrates. Trends Ecol. Evol. 22,

37 K.M. Fedorka et al., Immune suppression and the cost of reproduction in the ground cricket, Allonemobius socius, Evolution 58 (2004), pp. 24782485.

38 K.A. McKean and L. Nunney, Bateman's principle and immunity: phenotypically plastic reproductive strategies predict changes in immunological sex differences, Evolution 59 (2005), pp. 1510-1517.

39 J. Rolff and M.T. Siva-Jothy, Copulation corrupts immunity: a mechanism for a cost of mating in insects, Proc. Natl. Acad. Sci. U. S. A. 99 (2002), pp. 9916-9918.

$40 \mathrm{G}$. Amdam et al., Social reversal of immunosenescence in honey bee workers, Exp. Gerontol. 40 (2005), pp. 939-947.

41 S.A.O. Armitage et al., Examining costs of induced and constitutive immune investment in Tenebrio molitor, J. Evol. Biol. 16 (2003), pp. 10381044.

42 A. Jacot et al., Costs of an induced immune response on sexual display and longevity in field crickets, Evolution 58 (2004), pp. 2280-2286.

43 C. Gillot, Male accessory gland secretions: Modulators of female reproductive physiology and behavior, Annu. Rev. Entomol. 48 (2003), pp. 163-184.

44 M.F. Wolfner, Tokens of love: Functions and regulation of Drosophila male accessory gland products, Insect Biochem. Mol. Biol. 27 (1997) (3), pp. 179-192.

45 W.J. Swanson et al., Evolutionary EST analysis identifies rapidly evolving male reproductive proteins in Drosophila, Proc. Natl. Acad. Sci. U. S. A. 98 (2001), pp. 7375-7379.

46 T. Chapman et al., Cost of mating in Drosophila melanogaster females is mediated by male accessory gland products, Nature 373 (1995), pp. 241244.

47 O. Lung et al., The Drosophila melanogaster seminal fluid protein Acp62F is a protease inhibitor that is toxic upon ectopic expression, Genetics 160 (2002), pp. 211-224.

48 S. Wigby and T. Chapman, Sex peptide causes mating costs in female Drosophila melanogaster, Curr. Biol. 15 (2005), pp. 316-321.
49 J.S. Peng et al., Gradual release of sperm bound sex-peptide controls female postmating behavior in Drosophila, Curr. Biol. 15 (2005), pp. 207213.

50 P. Moshitzky et al., Sex-peptide activates juvenile hormone biosynthesis in the Drosophila melanogaster corpus allatum, Arch. Insect Biochem. Physiol. 32 (1996), pp. 363-374.

51 G.A. Parker, Sexual selection and sexual conflict. In: M.S. Blum and A.N. Blum, Editors, Sexual Selection and Reproductive Conflict in Insects, Academic Press (1979), pp. 123-166.

52 T. Tregenza et al., Sexual conflict: a new paradigm?, Phil. Trans. R. Soc. B 361 (2006), pp. 229-234.

53 N. Wedell et al., Sexual conflict and life histories, Anim. Behav. 71 (2006), pp. 999-1011.

54 L. Partridge and D. Gems, Beyond the evolutionary theory of ageing, from functional genomics to evo-gero, Trends Ecol. Evol. 21 (2006), pp. 334 340.

55 A.K. Chippindale et al., Complex trade-offs and the evolution of starvation resistance in Drosophila melanogaster, Evolution 50 (1996), pp. $753-766$.

56 A.B. Salmon et al., A cost of reproduction in Drosophila melanogaster: stress susceptibility, Evolution 55 (2001), pp. 1600-1608.

57 Y. Wang et al., A cost of reproduction: oxidative stress susceptibility is associated with increased egg production in Drosophila melanogaster, Exp. Gerontol. 36 (2001), pp. 1349-1359. Abstract

58 D. Harman, Ageing: a theory based on free radical and radiation chemistry, J. Gerontol. 11 (1956), pp. 298-300.

59 C. Alonso-Alvarez et al., Increased susceptibility to oxidative stress as a proximate cost of reproduction, Ecol. Lett. 7 (2004), pp. 363-368.

60 P. Wiersma et al., Birds sacrifice oxidative protection for reproduction, Proc. R. Soc. B 271 (2004) (Suppl. 5), pp. S360-S363.

$61 \mathrm{H}$. Hsin and C. Kenyon, Signals from the reproductive system regulate the lifespan of C. elegans, Nature 399 (1999), pp. 362-366.

62 J.H. Marden et al., Conditional tradeoffs between aging and organismal performance of INDY long-lived mutant flies, Proc. Natl. Acad. Sci. U. S. A. 100 (2003), pp. 3369-3373.

63 A. van Noordwijk and G. de Jong, Acquisition and allocation of resources: their influence on variation in life history tactics, Am. Nat. 128 (1986), pp. $137-142$.

64 L.G. Harshman, Genetic research on aging and longevity of Drosophila melanogaster, Popul. Dev. Rev. 29S (2003), pp. 99-126.

65 M. West-Eberhard, Developmental Plasticity and Evolution, Oxford University Press (2003).

66 R.Y. Lee et al., Regulation of C. elegans DAF-16 and its human ortholog FKHRL1 by the daf-2 insulin-like pathway, Curr. Biol. 11 (2001), pp. 1950-1957.

67 S.S. Lee et al., DAF-16 targets genes that control C. elegans life-span and metabolism, Science 300 (2003), pp. 644-647.

68 C.T. Murphy et al., Genes that act downstream of DAF-16 to influence the lifespan of Caenorhabditis elegans, Nature 424 (2003), pp. 277-284.

$69 \mathrm{~K}$. Jia et al., The TOR pathway interacts with the insulin signaling pathway to regulate C. elegans larval development, metabolism and lifespan, Development 131 (2004), pp. 3897-3906.

70 P. Karpathi and B. Zid, TOR pathway: linking nutrient sensing to lifespan, Sci. Aging Know. Environ. 36 (2004), pp. 885-890.

$71 \mathrm{~K}$. Lin et al., Regulation of the Caenorhabditis elegans longevity protein DAF-16 by insulin/IGF-1 and germline signaling, Nat. Genet. 28 (2001), pp. 139-145.

72 B. Rogina et al., Extended life-span conferred by cotransporter gene mutations in Drosophila, Science 290 (2000), pp. 2137-2140. 